\title{
Search for light sterile neutrinos with the T2K far detector Super-Kamiokande at a baseline of $295 \mathrm{~km}$
}

K. Abe, ${ }^{52}$ R. Akutsu ${ }^{53}$ A. Ali, ${ }^{23}$ C. Andreopoulos,${ }^{50,31}$ L. Anthony, ${ }^{31}$ M. Antonova, ${ }^{18}$ S. Aoki, ${ }^{28}$ A. Ariga, ${ }^{2}$ Y. Ashida, ${ }^{29}$ Y. Awataguchi, ${ }^{55}$ Y. Azuma, ${ }^{38}$ S. Ban ${ }^{29}$ M. Barbi ${ }^{42}$ G. J. Barker, ${ }^{62}$ G. Barr, ${ }^{39}$ C. Barry, ${ }^{31}$ M. Batkiewicz-Kwasniak, ${ }^{14}$ F. Bench ${ }^{31}$ V. Berardi, ${ }^{21}$ S. Berkman, ${ }^{4,58}$ R. M. Berner, ${ }^{2}$ L. Berns, ${ }^{54}$ S. Bhadra, ${ }^{66}$ S. Bienstock ${ }^{49}$ A. Blondel,,${ }^{12, \dagger}$ S. Bolognesi, ${ }^{6}$ B. Bourguille, ${ }^{17}$ S. B. Boyd ${ }^{62}$ D. Brailsford, ${ }^{30}$ A. Bravar, ${ }^{12}$ C. Bronner, ${ }^{52}$ M. Buizza Avanzini, ${ }^{10}$ J. Calcutt, ${ }^{33}$ T. Campbell, ${ }^{7}$ S. Cao, ${ }^{15}$ S. L. Cartwright ${ }^{46}$ M. G. Catanesi, ${ }^{21}$ A. Cervera ${ }^{18}$ A. Chappell, ${ }^{62}$ C. Checchia, ${ }^{23}$ D. Cherdack, ${ }^{16}$

N. Chikuma, ${ }^{51}$ G. Christodoulou, ${ }^{31,}$ J. Coleman, ${ }^{31}$ G. Collazuol, ${ }^{23}$ D. Coplowe,${ }^{39}$ A. Cudd, ${ }^{33}$ A. Dabrowska, ${ }^{14}$ G. De Rosa ${ }^{22}$ T. Dealtry,${ }^{30}$ P. F. Denner,${ }^{62}$ S. R. Dennis, ${ }^{31}$ C. Densham,${ }^{50}$ F. Di Lodovico,${ }^{41}$ N. Dokania, ${ }^{36}$ S. Dolan, ${ }^{10,6}$ O. Drapier, ${ }^{10}$ K. E. Duffy, ${ }^{39}$ J. Dumarchez ${ }^{49}$ P. Dunne, ${ }^{20}$ S. Emery-Schrenk, ${ }^{6}$ A. Ereditato, ${ }^{2}$ P. Fernandez, ${ }^{18}$ T. Feusels, ${ }^{4,58}$ A. J. Finch ${ }^{30}$ G. A. Fiorentini, ${ }^{66}$ G. Fiorillo, ${ }^{22}$ C. Francois, ${ }^{2}$ M. Friend, ${ }^{15}$ Y. Fujii, ${ }^{15,}$ R. Fujita, ${ }^{51}$ D. Fukuda ${ }^{37}$ Y. Fukuda, ${ }^{34}$ K. Gameil,${ }^{4,58}$ C. Giganti, ${ }^{49}$ F. Gizzarelli, ${ }^{6}$ T. Golan, ${ }^{64}$ M. Gonin, ${ }^{10}$ D. R. Hadley, ${ }^{62}$ J. T. Haigh,${ }^{62}$ P. Hamacher-Baumann, ${ }^{45}$ M. Hartz, ${ }^{58,27}$ T. Hasegawa, ${ }^{15,}$ N. C. Hastings, ${ }^{42}$ T. Hayashino, ${ }^{29}$ Y. Hayato, ${ }^{52,27}$ A. Hiramoto, ${ }^{29}$ M. Hogan, ${ }^{8}$ J. Holeczek ${ }^{47}$ N. T. Hong Van,${ }^{19,26}$ F. Hosomi,${ }^{51}$ F. Iacob, ${ }^{23}$ A. K. Ichikawa, ${ }^{29}$ M. Ikeda, ${ }^{52}$ T. Inoue, ${ }^{38}$ R. A. Intonti, ${ }^{21}$ T. Ishida, ${ }^{15,}$ T. Ishii, ${ }^{15, *}$ M. Ishitsuka, ${ }^{56}$ K. Iwamoto, ${ }^{51}$ A. Izmaylov, ${ }^{18,25}$ B. Jamieson, ${ }^{63}$ C. Jesus, ${ }^{17}$ M. Jiang,${ }^{29}$ S. Johnson, ${ }^{7}$ P. Jonsson, ${ }^{20}$ C. K. Jung, ${ }^{36,8}$ M. Kabirnezhad,${ }^{39}$ A. C. Kaboth,${ }^{4,50}$ T. Kajita ${ }^{53,8}$ H. Kakuno, ${ }^{55}$ J. Kameda, ${ }^{52}$ D. Karlen, ${ }^{59,58}$ T. Katori, ${ }^{41}$ Y. Kato, ${ }^{52}$ E. Kearns, ${ }^{3,27,8}$ M. Khabibullin, ${ }^{25}$ A. Khotjantsev, ${ }^{25}$ H. Kim, ${ }^{38}$ J. Kim, ${ }^{4,58}$ S. King, ${ }^{41}$ J. Kisiel, ${ }^{47}$ A. Knight, ${ }^{62}$ A. Knox, ${ }^{30}$ T. Kobayashi, ${ }^{15, \$}$ L. Koch,${ }^{50}$ T. Koga,${ }^{51}$ A. Konaka, ${ }^{58}$ L. L. Kormos, ${ }^{30}$ Y. Koshio, ${ }^{37,8}$ K. Kowalik,${ }^{35}$ H. Kubo, ${ }^{29}$ Y. Kudenko, ${ }^{25, \|}$ R. Kurjata, ${ }^{61}$ T. Kutter, ${ }^{32}$ M. Kuze,${ }^{54}$ L. Labarga, ${ }^{1}$ J. Lagoda, ${ }^{35}$ M. Lamoureux, ${ }^{6}$ P. Lasorak,${ }^{41}$ M. Laveder, ${ }^{23}$ M. Lawe, ${ }^{30}$ M. Licciardi, ${ }^{10}$ T. Lindner, ${ }^{58}$ R. P. Litchfield, ${ }^{13}$ X. Li ${ }^{36}$ A. Longhin, ${ }^{23}$ J. P. Lopez, ${ }^{7}$ T. Lou, ${ }^{51}$ L. Ludovici, ${ }^{24}$

X. Lu, ${ }^{39}$ T. Lux ${ }^{17}$ L. Magaletti, ${ }^{21}$ K. Mahn, ${ }^{33}$ M. Malek, ${ }^{46}$ S. Manly, ${ }^{43}$ L. Maret, ${ }^{12}$ A. D. Marino, ${ }^{7}$ J. F. Martin, ${ }^{57}$

P. Martins,${ }^{41}$ T. Maruyama, ${ }^{15,}$ T. Matsubara, ${ }^{15}$ V. Matveev,${ }^{25}$ K. Mavrokoridis, ${ }^{31}$ W. Y. Ma, ${ }^{20}$ E. Mazzucato, ${ }^{6}$

M. McCarthy, ${ }^{66}$ N. McCauley, ${ }^{31}$ K. S. McFarland, ${ }^{43}$ C. McGrew,${ }^{36}$ A. Mefodiev, ${ }^{25}$ C. Metelko, ${ }^{31}$ M. Mezzetto ${ }^{23}$

A. Minamino, ${ }^{65}$ O. Mineev ${ }^{25}$ S. Mine,${ }^{5}$ M. Miura ${ }^{52,8}$ L. Molina Bueno, ${ }^{11}$ S. Moriyama ${ }^{52,}$ J. Morrison, ${ }^{33}$ Th. A. Mueller, ${ }^{10}$

S. Murphy ${ }_{11}^{11}$ Y. Nagai, ${ }^{7}$ T. Nakadaira, ${ }^{15,}$ M. Nakahata ${ }^{52,27}$ Y. Nakajima, ${ }^{52}$ A. Nakamura, ${ }^{37}$ K. G. Nakamura, ${ }^{29}$

K. Nakamura, ${ }^{27,15, *}$ K. D. Nakamura, ${ }^{29}$ Y. Nakanishi, ${ }^{29}$ S. Nakayama, ${ }^{52,8}$ T. Nakaya, ${ }^{29,27}$ K. Nakayoshi,${ }^{15, *}$ C. Nantais ${ }^{57}$ K. Niewczas, ${ }^{64}$ K. Nishikawa, ${ }^{15 *}$ Y. Nishimura, ${ }^{53}$ T. S. Nonnenmacher ${ }^{20}$ P. Novella, ${ }^{18}$ J. Nowak, ${ }^{30}$ H. M. O' Keeffe, ${ }^{30}$ L. O'Sullivan, ${ }^{46}$ K. Okumura, ${ }^{53,27}$ T. Okusawa, ${ }^{38}$ S. M. Oser, ${ }^{4,58}$ R. A. Owen, ${ }^{41}$ Y. Oyama, ${ }^{15,}$ V. Palladino, ${ }^{22}$ J. L. Palomino, ${ }^{36}$ V. Paolone, ${ }^{40}$ W. C. Parker, ${ }^{44}$ P. Paudyal,${ }^{31}$ M. Pavin, ${ }^{58}$ D. Payne, ${ }^{31}$ L. Pickering, ${ }^{33}$ C. Pidcott, ${ }^{46}$ E. S. Pinzon Guerra ${ }^{66}$ C. Pistillo, ${ }^{2}$ B. Popov, ${ }^{49,}$ K. Porwit, ${ }^{47}$ M. Posiadala-Zezula, ${ }^{60}$ A. Pritchard, ${ }^{31}$ B. Quilain, ${ }^{27}$ T. Radermacher, ${ }^{45}$ E. Radicioni, ${ }^{21}$ B. Radics, ${ }^{11}$ P. N. Ratoff, ${ }^{30}$ E. Reinherz-Aronis, ${ }^{8}$ C. Riccio, ${ }^{22}$ E. Rondio,${ }^{35}$ B. Rossi ${ }^{22}$ S. Roth ${ }^{45}$ A. Rubbia, ${ }^{11}$ A. C. Ruggeri, ${ }^{22}$ A. Rychter, ${ }^{61}$ K. Sakashita ${ }^{15, \$}$ F. Sánchez, ${ }^{12}$ S. Sasaki, ${ }^{55}$ C. M. Schloesser ${ }^{11}$ K. Scholberg,,${ }^{9,8}$ J. Schwehr, ${ }^{8}$ M. Scott ${ }^{20}$ Y. Seiya, ${ }^{38}$ T. Sekiguchi, ${ }^{15,}$ H. Sekiya, ${ }^{52,27,8}$ D. Sgalaberna, ${ }^{12}$ R. Shah, ${ }^{50,39}$ A. Shaikhiev, ${ }^{25}$ F. Shaker, ${ }^{63}$ D. Shaw, ${ }^{30}$ A. Shaykina, ${ }^{25}$ M. Shiozawa, ${ }^{52,27}$ A. Smirnov, ${ }^{25}$ M. Smy, ${ }^{5}$ J. T. Sobczyk ${ }^{64}$ H. Sobel, ${ }^{5,27}$ Y. Sonoda, ${ }^{52}$ J. Steinmann, ${ }^{45}$ T. Stewart, ${ }^{50}$ P. Stowell, ${ }^{46}$ S. Suvorov,${ }^{25,6}$ A. Suzuki, ${ }^{28}$ S. Y. Suzuki, ${ }^{15,}$ Y. Suzuki, ${ }^{27}$ A. A. Sztuc, ${ }^{20}$ R. Tacik,${ }^{42,58}$ M. Tada ${ }^{15, \$}$ A. Takeda,${ }^{52}$ Y. Takeuchi,${ }^{28,27}$ R. Tamura, ${ }^{51}$ H. K. Tanaka, ${ }^{52,}$ H. A. Tanaka, ${ }^{48,57}$ L. F. Thompson ${ }^{46}$ W. Toki,${ }^{8}$ C. Touramanis,${ }^{31}$ K. M. Tsui, ${ }^{31}$ T. Tsukamoto, ${ }^{15}$ M. Tzanov,${ }^{32}$ Y. Uchida ${ }^{20}$

W. Uno, ${ }^{29}$ M. Vagins, ${ }^{27,5}$ Z. Vallari, ${ }^{36}$ D. Vargas, ${ }^{17}$ G. Vasseur, ${ }^{6}$ C. Vilela, ${ }^{36}$ T. Vladisavljevic, ${ }^{39,27}$ V. V. Volkov, ${ }^{25}$

T. Wachala, ${ }^{14}$ J. Walker, ${ }^{63}$ Y. Wang, ${ }^{36}$ D. Wark, ${ }^{50,39}$ M. O. Wascko, ${ }^{20}$ A. Weber, ${ }^{50,39}$ R. Wendell,,${ }^{29,}$ M. J. Wilking, ${ }^{36}$ C. Wilkinson, ${ }^{2}$ J. R. Wilson, ${ }^{41}$ R. J. Wilson, ${ }^{8}$ C. Wret, ${ }^{43}$ Y. Yamada, ${ }^{15, *}$ K. Yamamoto, ${ }^{38}$ S. Yamasu, ${ }^{37}$ C. Yanagisawa, ${ }^{36, * *}$ G. Yang, ${ }^{36}$ T. Yano,${ }^{52}$ K. Yasutome, ${ }^{29}$ S. Yen, ${ }^{58}$ N. Yershov, ${ }^{25}$ M. Yokoyama, ${ }^{51,8}$ T. Yoshida, ${ }^{54}$ M. Yu,${ }^{66}$ A. Zalewska, ${ }^{14}$ J. Zalipska, ${ }^{35}$ K. Zaremba, ${ }^{61}$ G. Zarnecki, ${ }^{35}$ M. Ziembicki, ${ }^{61}$ E. D. Zimmerman, ${ }^{7}$ M. Zito, ${ }^{6}$ S. Zsoldos, ${ }^{41}$ and A. Zykova ${ }^{25}$

(T2K Collaboration)

\footnotetext{
${ }^{1}$ University Autonoma Madrid, Department of Theoretical Physics, 28049, Madrid, Spain

${ }^{2}$ University of Bern, Albert Einstein Center for Fundamental Physics, Laboratory for High Energy Physics (LHEP), Bern, Switzerland

${ }^{3}$ Boston University, Department of Physics, Boston, Massachusetts, USA

${ }^{4}$ University of British Columbia, Department of Physics and Astronomy, Vancouver, British Columbia, Canada

${ }^{5}$ University of California, Irvine, Department of Physics and Astronomy, Irvine, California, USA ${ }^{6} I R F U, C E A$ Saclay, Gif-sur-Yvette, France
} 
${ }^{7}$ University of Colorado at Boulder, Department of Physics, Boulder, Colorado, USA

${ }^{8}$ Colorado State University, Department of Physics, Fort Collins, Colorado, USA

${ }^{9}$ Duke University, Department of Physics, Durham, North Carolina, USA

${ }^{10}$ Ecole Polytechnique, IN2P3-CNRS, Laboratoire Leprince-Ringuet, Palaiseau, France

${ }^{11}$ ETH Zurich, Institute for Particle Physics, Zurich, Switzerland

${ }^{12}$ University of Geneva, Section de Physique, DPNC, Geneva, Switzerland

${ }^{13}$ University of Glasgow, School of Physics and Astronomy, Glasgow, United Kingdom

${ }^{14} \mathrm{H}$. Niewodniczanski Institute of Nuclear Physics PAN, Cracow, Poland

${ }^{15}$ High Energy Accelerator Research Organization (KEK), Tsukuba, Ibaraki, Japan

${ }^{16}$ University of Houston, Department of Physics, Houston, Texas, USA

${ }^{17}$ Institut de Fisica d'Altes Energies (IFAE), The Barcelona Institute of Science and Technology, Campus $U A B$, Bellaterra (Barcelona), Spain

${ }^{18}$ IFIC (CSIC \& University of Valencia), Valencia, Spain

${ }^{19}$ Institute For Interdisciplinary Research in Science and Education (IFIRSE), ICISE, Quy Nhon, Vietnam

${ }^{20}$ Imperial College London, Department of Physics, London, United Kingdom

${ }^{21}$ INFN Sezione di Bari and Università e Politecnico di Bari, Dipartimento Interuniversitario di Fisica, Bari, Italy

${ }^{22}$ INFN Sezione di Napoli and Università di Napoli, Dipartimento di Fisica, Napoli, Italy

${ }^{23}$ INFN Sezione di Padova and Università di Padova, Dipartimento di Fisica, Padova, Italy

${ }^{24}$ INFN Sezione di Roma and Università di Roma "La Sapienza," Roma, Italy

${ }^{25}$ Institute for Nuclear Research of the Russian Academy of Sciences, Moscow, Russia

${ }^{26}$ Institute of Physics (IOP), Vietnam Academy of Science and Technology (VAST), Hanoi, Vietnam

${ }^{27}$ Kavli Institute for the Physics and Mathematics of the Universe (WPI),

The University of Tokyo Institutes for Advanced Study, University of Tokyo, Kashiwa, Chiba, Japan

${ }^{28}$ Kobe University, Kobe, Japan

${ }^{29}$ Kyoto University, Department of Physics, Kyoto, Japan

${ }^{30}$ Lancaster University, Physics Department, Lancaster, United Kingdom

${ }^{31}$ University of Liverpool, Department of Physics, Liverpool, United Kingdom

${ }^{32}$ Louisiana State University, Department of Physics and Astronomy, Baton Rouge, Louisiana, USA

${ }^{33}$ Michigan State University, Department of Physics and Astronomy, East Lansing, Michigan, USA

${ }^{34}$ Miyagi University of Education, Department of Physics, Sendai, Japan

${ }^{35}$ National Centre for Nuclear Research, Warsaw, Poland

${ }^{36}$ State University of New York at Stony Brook, Department of Physics and Astronomy, Stony Brook, New York, USA

${ }^{37}$ Okayama University, Department of Physics, Okayama, Japan

${ }^{38}$ Osaka City University, Department of Physics, Osaka, Japan

${ }^{39}$ Oxford University, Department of Physics, Oxford, United Kingdom

${ }^{40}$ University of Pittsburgh, Department of Physics and Astronomy, Pittsburgh, Pennsylvania, USA

${ }^{41}$ Queen Mary University of London, School of Physics and Astronomy, London, United Kingdom

${ }^{42}$ University of Regina, Department of Physics, Regina, Saskatchewan, Canada

${ }^{43}$ University of Rochester, Department of Physics and Astronomy, Rochester, New York, USA

${ }^{44}$ Royal Holloway University of London, Department of Physics, Egham, Surrey, United Kingdom

${ }^{45}$ RWTH Aachen University, III. Physikalisches Institut, Aachen, Germany

${ }^{46}$ University of Sheffield, Department of Physics and Astronomy, Sheffield, United Kingdom

${ }^{47}$ University of Silesia, Institute of Physics, Katowice, Poland

${ }^{48}$ SLAC National Accelerator Laboratory, Stanford University, Menlo Park, California, USA

${ }^{49}$ Sorbonne Université, Université Paris Diderot, CNRS/IN2P3,

Laboratoire de Physique Nucléaire et de Hautes Energies (LPNHE), Paris, France

${ }^{50}$ STFC, Rutherford Appleton Laboratory, Harwell Oxford, and Daresbury Laboratory,

Warrington, United Kingdom

${ }^{51}$ University of Tokyo, Department of Physics, Tokyo, Japan

${ }^{52}$ University of Tokyo, Institute for Cosmic Ray Research, Kamioka Observatory, Kamioka, Japan

${ }^{53}$ University of Tokyo, Institute for Cosmic Ray Research,

Research Center for Cosmic Neutrinos, Kashiwa, Japan

${ }^{54}$ Tokyo Institute of Technology, Department of Physics, Tokyo, Japan

${ }^{55}$ Tokyo Metropolitan University, Department of Physics, Tokyo, Japan

${ }^{56}$ Tokyo University of Science, Faculty of Science and Technology, Department of Physics, Noda, Chiba, Japan

${ }^{57}$ University of Toronto, Department of Physics, Toronto, Ontario, Canada

${ }^{58}$ TRIUMF, Vancouver, British Columbia, Canada 
${ }^{59}$ University of Victoria, Department of Physics and Astronomy, Victoria, British Columbia, Canada

${ }^{60}$ University of Warsaw, Faculty of Physics, Warsaw, Poland

${ }^{61}$ Warsaw University of Technology, Institute of Radioelectronics, Warsaw, Poland

${ }^{62}$ University of Warwick, Department of Physics, Coventry, United Kingdom

${ }^{63}$ University of Winnipeg, Department of Physics, Winnipeg, Manitoba, Canada

${ }^{64}$ Wroclaw University, Faculty of Physics and Astronomy, Wroclaw, Poland

${ }^{65}$ Yokohama National University, Faculty of Engineering, Yokohama, Japan

${ }^{66}$ York University, Department of Physics and Astronomy, Toronto, Ontario, Canada

(Received 19 February 2019; published 30 April 2019; corrected 7 June 2019)

\begin{abstract}
We perform a search for light sterile neutrinos using the data from the T2K far detector at a baseline of $295 \mathrm{~km}$, with an exposure of $14.7(7.6) \times 10^{20}$ protons on target in neutrino (antineutrino) mode. A selection of neutral-current interaction samples is also used to enhance the sensitivity to sterile mixing. No evidence of sterile neutrino mixing in the $3+1$ model was found from a simultaneous fit to the charged-current muon, electron and neutral-current neutrino samples. We set the most stringent limit on the sterile oscillation amplitude $\sin ^{2} \theta_{24}$ for the sterile neutrino mass splitting $\Delta m_{41}^{2}<3 \times 10^{-3} \mathrm{eV}^{2} / c^{4}$.
\end{abstract}

DOI: 10.1103/PhysRevD.99.071103

\section{INTRODUCTION}

Over the last few decades, the theory of neutrino oscillations has been well established through a series of experiments with neutrinos produced by the Sun [1-6], nuclear reactors [7-10], accelerators [11-15] and in the atmosphere [16,17]. Most data from these studies are consistent with the three flavor paradigm where the three weakly interacting neutrino flavors are related to three neutrino mass states by the Pontecorvo-Maki-NakagawaSakata mixing matrix [18-20]. However, deviations from the three flavor scheme have been reported. At LSND [21] and MiniBooNE [22], there were excesses of $\bar{\nu}_{e}$ found in short-baseline $\bar{\nu}_{\mu}$ beams; MiniBooNE also reported an excess in $\nu_{e}$ appearance [23]; radioactive calibration sources in gallium experiments [24,25] showed a deficit of $\nu_{e}$ flux; and reactor experiments [26] observed less $\bar{\nu}_{e}$ than expected. These results could be explained by a fourth neutrino state with a mass difference $\Delta m^{2} \sim 1 \mathrm{eV}^{2} / c^{4}$ with respect to the three Pontecorvo-Maki-Nakagawa-Sakata states [27-30]. From the measurements of the invisible decay width of the $Z^{0}$ boson at the LEP collider, the number of weakly interacting neutrino species with mass below

\footnotetext{
*Deceased.

†Present address: CERN, Geneva, Switzerland.

${ }^{\ddagger}$ Also at J-PARC, Tokai, Japan.

${ }^{\S}$ Also at Kavli IPMU (WPI), the University of Tokyo, Japan.

"Also at National Research Nuclear University "MEPhI" and Moscow Institute of Physics and Technology, Moscow, Russia. "Also at JINR, Dubna, Russia.

** Also at BMCC/CUNY, Science Department, New York, New York, USA.

Published by the American Physical Society under the terms of the Creative Commons Attribution 4.0 International license. Further distribution of this work must maintain attribution to the author(s) and the published article's title, journal citation, and DOI. Funded by SCOAP.
}

$45 \mathrm{GeV} / c^{2}$ is limited to three [31], so the new neutrino state must not couple to the weak interaction and is often referred to as "sterile." We can incorporate this additional neutrino state in the simple " $3+1$ " model [28], which involves the three active neutrinos and one sterile neutrino, and study its effect on the oscillation signatures. Currently, the null results, especially in the $\stackrel{(-)}{\nu}_{\mu}$ disappearance channels, from short-baseline accelerator experiments like CCFR [32], MiniBooNE/SciBooNE [33] and T2K [34]; long-baseline experiments like MINOS and MINOS+ [35] and $\mathrm{NO} \nu \mathrm{A}$ [36]; or atmospheric experiments like SuperKamiokande [37] and IceCube [38,39], have limited the available parameter space in the " $3+1$ " model.

The Tokai to Kamioka (T2K) experiment is a longbaseline accelerator neutrino experiment in Japan which primarily measures muon neutrino disappearance and electron neutrino appearance. While $\mathrm{T} 2 \mathrm{~K}$ is designed for studying standard three flavor oscillation at $\Delta m^{2} \sim$ $10^{-3} \mathrm{eV}^{2} / c^{4}$, it also has the potential to search for oscillation signatures due to sterile neutrinos around this $\Delta m^{2}$ range. Neutral-current (NC) neutrino interactions are also collected in the far detector, Super-Kamiokande (SK), which can be used to enhance the sensitivity to sterile mixing as the sterile neutrinos, unlike other active neutrinos, do not interact through $\mathrm{CC}$ or $\mathrm{NC}$ scattering. We present a long-baseline search for sterile neutrinos in the " $3+1$ " framework, using both the charged-current $(\mathrm{CC}) \stackrel{(-)}{\nu}_{\mu}$ and $\stackrel{(-)}{\nu}_{e}$ samples and $\mathrm{NC}$ samples at the far detector.

Section II briefly describes the sterile neutrino mixing model and its effect on the oscillation probability (or oscillation signatures). The $\mathrm{T} 2 \mathrm{~K}$ experimental setup is outlined in Sec. III, followed by event selection criteria in Sec. IV. Section V explains the analysis strategy and Sec. VI presents our search results. Finally, Sec. VII gives a summary and outlook of our sterile neutrino study. 


\section{II. “3 + 1” STERILE NEUTRINO MIXING}

In this study, we focus on a " $3+1$ "-like model where a single sterile neutrino is added and mixed with the three active states, which is the simplest model with a sterile neutrino frequently used in neutrino oscillation analysis. In this model, there is a new flavor state $\nu_{s}$ and a new mass state $\nu_{4}$ with mass $m_{4}$ added to the three flavor framework. The relation between the flavor and mass states is given by

$$
\left|\nu_{\alpha}\right\rangle=\sum U_{\alpha k}^{*}\left|\nu_{k}\right\rangle
$$

where $\left|\nu_{\alpha}\right\rangle$ are the flavor states and $\left|\nu_{k}\right\rangle$ are the mass states. The original $3 \times 3$ Pontecorvo-Maki-Nakagawa-Sakata mixing matrix is expanded to a $4 \times 4$ matrix as

$$
\mathbf{U}=\left(\begin{array}{cccc}
U_{e 1} & U_{e 2} & U_{e 3} & U_{e 4} \\
U_{\mu 1} & U_{\mu 2} & U_{\mu 3} & U_{\mu 4} \\
U_{\tau 1} & U_{\tau 2} & U_{\tau 3} & U_{\tau 4} \\
U_{s 1} & U_{s 2} & U_{s 3} & U_{s 4}
\end{array}\right)
$$

We choose the parametrization as in [40]:

$$
\mathbf{U}=\mathbf{U}_{34} \mathbf{U}_{24} \mathbf{U}_{14} \mathbf{U}_{23} \mathbf{U}_{13} \mathbf{U}_{12},
$$

where $\mathbf{U}_{i j}$ is a unitary rotation matrix of an angle $\theta_{i j}$ in the $i j$-plane. There are therefore three new mixing angles $\theta_{14}$, $\theta_{24}, \theta_{34}$ and two new $C P$-violating phases $\delta_{14}, \delta_{24}$. Note that $\sin ^{2} \theta_{14}$ has been constrained to small values by reactor experiments [41], and T2K has limited sensitivity to $\theta_{14}$ and the new $C P$ phases. Since there is no significant correlation between them and the other oscillation parameters in this study, we set $\theta_{14}=\delta_{14}=\delta_{24}=0$ to simplify the mixing matrix.

At the far detector, the $\nu_{\mu}$ survival probability can be approximated (omitting $\delta_{C P}$ terms) as

$$
\begin{aligned}
P\left(\nu_{\mu} \rightarrow \nu_{\mu}\right) \approx & 1-\sin ^{2} 2 \theta_{23} \cos ^{4} \theta_{24} \sin ^{2} \frac{\Delta m_{31}^{2} L}{4 E} \\
& -\cos ^{2} \theta_{23} \sin ^{2} 2 \theta_{24} \sin ^{2} \frac{\Delta m_{41}^{2} L}{4 E} \\
& -\sin ^{2} \theta_{23} \sin ^{2} 2 \theta_{24} \sin ^{2} \frac{\Delta m_{43}^{2} L}{4 E}
\end{aligned}
$$

and the $\nu_{e}$ appearance probability as

$$
P\left(\nu_{\mu} \rightarrow \nu_{e}\right) \approx \sin ^{2} 2 \theta_{13} \cos ^{2} \theta_{24} \sin ^{2} \theta_{23} \sin ^{2} \frac{\Delta m_{31}^{2} L}{4 E} .
$$

Thus the CC channels are sensitive to $\theta_{24}$ and $\Delta m_{41}^{2}$. Similarly, the active neutrino survival probability, which is manifested in the NC channel, is sensitive to $\theta_{24}, \Delta m_{41}^{2}$, and $\theta_{34}$ :

$$
\begin{aligned}
P_{\mathrm{NC}}= & 1-P\left(\nu_{\mu} \rightarrow \nu_{s}\right) \\
\approx & 1-\sin ^{2} 2 \theta_{23}\left(A^{2}-\frac{1}{4} B^{2}\right) \sin ^{2} \frac{\Delta m_{31}^{2} L}{4 E} \\
& -B\left(B \cos ^{2} \theta_{23}-A \sin 2 \theta_{23}\right) \sin ^{2} \frac{\Delta m_{41}^{2} L}{4 E} \\
& -B\left(B \sin ^{2} \theta_{23}+A \sin 2 \theta_{23}\right) \sin ^{2} \frac{\Delta m_{43}^{2} L}{4 E},
\end{aligned}
$$

where $A=\cos \theta_{24} \sin \theta_{34}$ and $B=\sin 2 \theta_{24} \cos \theta_{34}$. The antineutrino oscillation probabilities follow similarly, but there are small differences due to the $\delta_{C P}$ terms which are not explicitly written here. Figure 1 shows schematically how the oscillation probabilities are modified with the mixing of sterile neutrinos.

The addition of a sterile neutrino state which experiences no matter potential (both $\mathrm{CC}$ and $\mathrm{NC}$ ) complicates the calculation of oscillation in matter. We estimated the possible impacts due to matter effects at $\mathrm{T} 2 \mathrm{~K}$ baseline and energy with a numerical calculation of oscillation probabilities using nuCraft [42], and we found at most a few percent changes for the $\stackrel{(-)}{\nu}$ and NC samples. This is negligible at current precision, so we simply use the vacuum oscillation probabilities (without approximation) in this study.
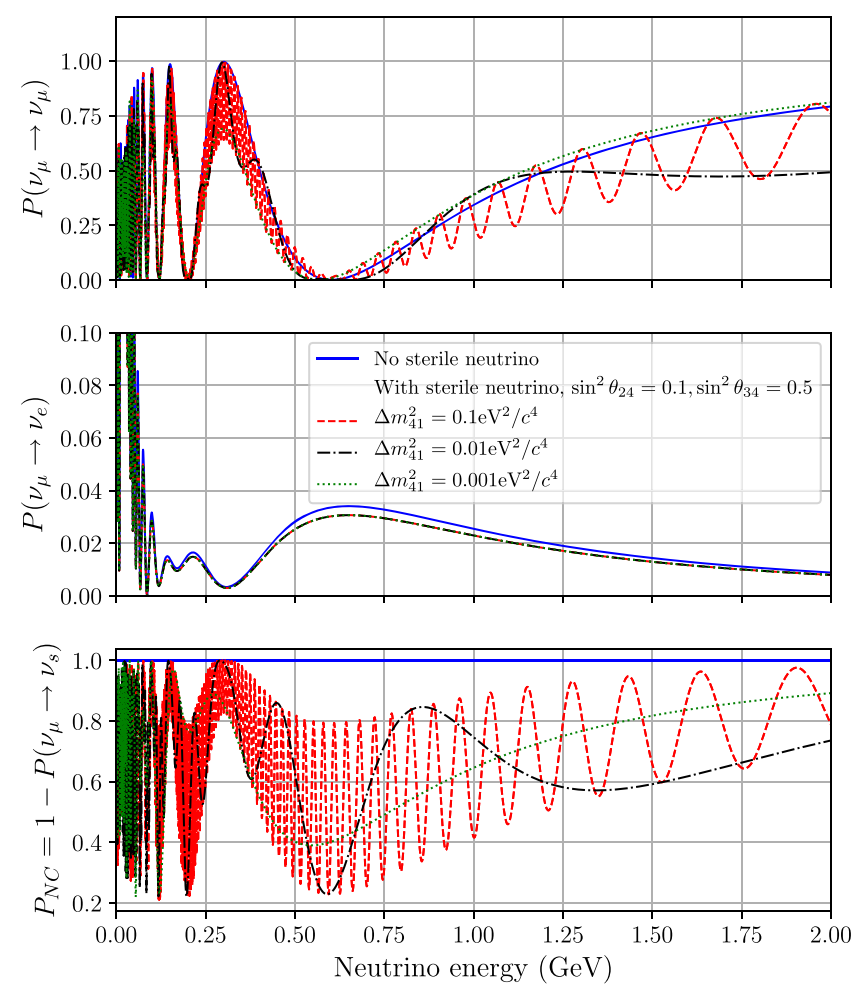

FIG. 1. The muon neutrino survival probability (top), electron neutrino appearance probability (middle) and active neutrino survival probability (bottom) as a function of neutrino energy, with and without a sterile neutrino, assuming $\Delta m_{31}^{2}>0$. 


\section{THE T2K EXPERIMENT}

The T2K experiment [43] consists of a neutrino beam, a near detector complex, and the water Cherenkov detector Super-Kamiokande [44] as the far detector at a baseline of $295 \mathrm{~km} . \mathrm{T} 2 \mathrm{~K}$ is sensitive to $\theta_{13}$ and $\delta_{C P}$ through the $\stackrel{(-)}{\nu}{ }_{e}$ appearance searches, and can also make precision measurements on $\theta_{23}$ and mass difference $\Delta m_{32}^{2}$ with the $\stackrel{(-)}{\nu}_{\mu}$ disappearance samples.

The main ring accelerator in the Japan Proton Accelerator Research Complex (J-PARC) produces a $30 \mathrm{GeV}$ proton beam with spills every $2.48 \mathrm{~s}$ that contain eight beam bunches which are $580 \mathrm{~ns}$ apart. The proton beam is extracted to the neutrino beam line and collides with a graphite target to produce charged pions and kaons. In the neutrino (antineutrino) beam mode, magnetic horns are used to focus the positively (negatively) charged pions and kaons into a $96 \mathrm{~m}$ long decay volume filled with helium gas. These mesons typically decay into muon neutrinos (antineutrinos). The neutrino beam line is directed at an angle of $2.5^{\circ}$ away from the far detector, so that the off-axis beam at SK has a narrower peak at $0.6 \mathrm{GeV}$ with much less $\stackrel{(-)}{\nu}$ e content than an on-axis beam.

The near detector complex located $280 \mathrm{~m}$ from the neutrino production target contains two detectors. The onaxis detector, INGRID, is an array of 16 iron/scintillator detectors which precisely measure the beam direction and intensity [45]. The off-axis detector, ND280, is a magnetized tracking detector which constrains the neutrino flux and cross-section model parameters in the oscillation analysis.

The far detector, Super-Kamiokande, is located in Gifu prefecture, at a distance of $295 \mathrm{~km}$ from the J-PARC neutrino beam. It is a water Cherenkov detector consisting of $50 \mathrm{kt}$ of ultrapure water. The tank is optically separated into two regions. The inner detector (ID) is a cylinder containing $32 \mathrm{kt}$ of water and is instrumented with 11,129 inward-facing 20 inch photomultiplier tubes (PMTs). The outer detector (OD) extends $2 \mathrm{~m}$ outward from the ID and is instrumented with 1885 outward-facing 8 inch PMTs. The OD serves as an active veto against cosmic-ray muons and provides passive shielding from radioactivity in the surrounding rock. The expected event rates at the far detector are calculated by multiplying the unoscillated neutrino spectra (predicted by near detectors) with the corresponding oscillation probabilities.

\section{EVENT SELECTION AT FAR DETECTOR}

The T2K Runs 1-8 data set used in this analysis was collected from January 2010 to May 2017, corresponding to a beam exposure of $14.7 \times 10^{20}$ protons on target (POT) in neutrino mode and $7.6 \times 10^{20} \mathrm{POT}$ in antineutrino mode. Events at the far detector are required to occur within $1 \mathrm{~ms}$ of the beam spill time window and to be fully contained in the fiducial volume of the SK ID. For the CC $\stackrel{(-)}{\nu}_{\mu}$ and $\stackrel{(-)}{\nu}_{e}$ samples, a new Cherenkov-ring reconstruction algorithm [46] is used to select neutrino events, which improves signal/background discrimination and expands the fiducial volume to increase statistics.

There are five CC analysis samples that are commonly used in the standard three flavor oscillation analysis [47]: $\stackrel{(-)}{\nu}, \mathrm{CC}-0 \pi$ and $\stackrel{(-)}{\nu}) \mathrm{CC}-0 \pi$ samples which are enriched in $\mathrm{CC}$ quasielastic (CCQE) events, and a $\nu_{e} \mathrm{CC}-1 \pi^{+}$sample where a $\pi^{+}$below the Cherenkov threshold is produced. The $\stackrel{(-)}{\nu}_{\mu}$ samples are binned in reconstructed neutrino energy and the $\stackrel{(-)}{\nu}$ samples in reconstructed lepton momentum and angle $\theta$ relative to the beam. Details can be found in [47]. Table I summarizes the event rates, where the Monte Carlo (MC) expectation is calculated with $\sin ^{2} \theta_{23}=0.528, \quad \Delta m_{32}^{2}=2.509 \times 10^{-3} \mathrm{eV}^{2} / c^{4}, \quad \delta_{C P}=$ -1.601 (the most probable values obtained by the Bayesian analysis in [46]), and $\sin ^{2} \theta_{13}=0.0219$ (taken from [48]). All sterile mixing angles are set to zero.

In addition to the $\mathrm{CC}$ samples, beam-induced $\mathrm{NC}$ events are also collected in SK. These events have previously only been used in publications for systematic uncertainties [47] and cross-section [49] studies. In this analysis, $\mathrm{NC} \pi^{0}$ and $\mathrm{NC} \gamma$-deexcitation samples are used in the oscillation fit to enhance the sensitivity to sterile mixing parameters.

The $\mathrm{NC} \pi^{0}$ samples select neutrino events with single $\pi^{0}$ production, where $\pi^{0} \rightarrow 2 \gamma$ decay produces two visible Cherenkov rings in the detector. Events with two electronlike Cherenkov rings are selected as candidates for $\mathrm{NC} \pi^{0}$ samples, and those with decay electron candidates (from muons) are rejected. The invariant mass from the two rings is required to be between 85 and $135 \mathrm{MeV} / c^{2}$ to be consistent with the $\pi^{0}$ mass. From simulations, $68.5 \%$ (53.6\%) of events originate from a $\Delta$ resonance and $19.1 \%$ (34.9\%) from coherent pion production for the (anti) neutrino mode. The $\mathrm{NC}$ single pion resonant $(\mathrm{NC} 1 \pi)$ production is described by the Rein-Sehgal model [50],

TABLE I. Number of events expected and observed in the eight oscillation samples used in this analysis. Three flavor oscillation is assumed in expected rate.

\begin{tabular}{lcc}
\hline \hline Sample & Expected & Observed \\
\hline$\nu_{\mu}$ CC-0 $\pi$ & 268.4 & 240 \\
$\bar{\nu}_{\mu}$ CC-0 $\pi$ & 64.3 & 68 \\
$\nu_{e}$ CC-0 $\pi$ & 73.5 & 74 \\
$\bar{\nu}_{e}$ CC- $0 \pi$ & 7.9 & 7 \\
$\nu_{e}$ CC- $1 \pi^{+}$ & 6.9 & 15 \\
$\nu$ NC $\pi^{0}$ & 49.5 & 53 \\
$\bar{\nu}$ NC $\pi^{0}$ & 11.3 & 9 \\
NC $\gamma$-deexcit. & 107.7 & 102 \\
(Runs 1-4) & & \\
\hline \hline
\end{tabular}


while the coherent production is described by a tuned model of Rein-Sehgal [51]. In MC, NC events constitute $97.1 \%(98.5 \%)$ of the sample.

The NC $\gamma$-deexcitation sample was first reported in the measurement of the neutrino-oxygen $\mathrm{NC}$ quasielastic (NCQE) cross section [49]. The NCQE cross section is calculated by a spectral function model $[52,53]$ with the BBBA05 form factor parametrization [54], reweighting as a function of neutrino energy to match the theoretical calculations [55]. The NCQE interaction can knock out a nucleon,

$\nu+{ }^{16} \mathrm{O} \rightarrow \nu+p+{ }^{15} \mathrm{~N}^{*}, \quad$ or $\quad \nu+{ }^{16} \mathrm{O} \rightarrow \nu+n+{ }^{15} \mathrm{O}^{*}$,

which produces primary $\gamma$ rays from residual nucleus deexcitation and secondary $\gamma$ rays when knocked-out nucleons interact with other nuclei in water. The emitted $\gamma$ rays are $10 \mathrm{MeV}$ per event on average, which is much less energetic than other samples. Momenta and vertex positions are reconstructed using the low-energy tools developed for SK solar neutrino analyses [56]. Cuts on reconstructed energy, fiducial volume, event timing, vertex and reconstruction quality, detector preactivity, and Cherenkov opening angle are applied sequentially to remove beam-unrelated (e.g., radioactivity) and beamrelated $\mathrm{CC}$ backgrounds. The sample is estimated to contain $76.9 \%$ NCQE and $17.6 \%$ NC non-QE events. The NC $\gamma$-deexcitation sample is currently available only for T2K Runs 1-4 (from January 2010 to May 2013), corresponding to $6.56 \times 10^{20} \mathrm{POT}$. The remaining data are under reduction and validation with an improved event selection process.

Figure 2 shows the reconstructed $\pi^{0}$ momentum and $\gamma$ energy distributions of the $\mathrm{NC} \pi^{0}$ and $\mathrm{NC} \gamma$-deexcitation samples respectively, with event rates summarized in Table I. Since the event spectra have little information about true neutrino energy, the sensitivity to $\Delta m_{41}^{2}$ is limited in the NC channel.

\section{ANALYSIS STRATEGY}

The overall analysis method is similar to that used in the standard T2K three flavor analysis [47], including the incorporation of off-axis near detector data and treatment of systematic uncertainties. However, this analysis performs a simultaneous fit to the five CC and three newly added NC samples to constrain the sterile mixing parameters in the " $3+1$ " model. Systematic parameters are added to accommodate the possible uncertainties in the $\mathrm{NC}$ samples.

The neutrino flux is calculated by a data-driven simulation [57,58], which incorporates the conditions of the proton beam, magnetic horn current and neutrino beamaxis direction. Hadronic interactions are tuned with the thin target measurements in the NA61/SHINE experiment [59]. Stability of the neutrino flux has been monitored by
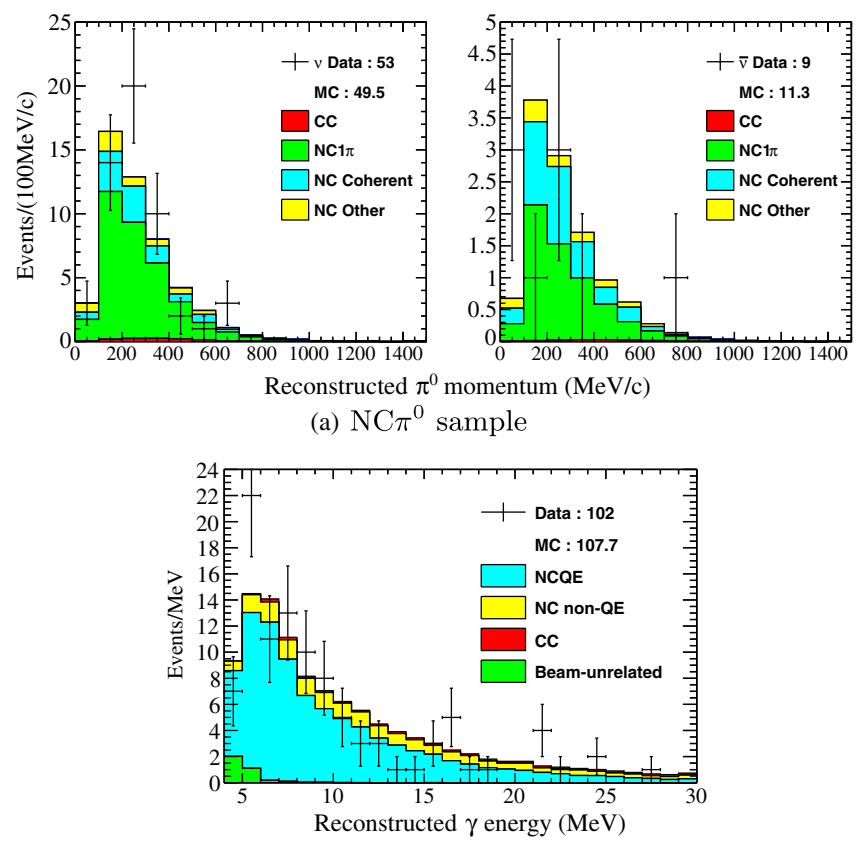

(b) $\mathrm{NC} \gamma$-deexcitation sample (Runs 1-4)

FIG. 2. Reconstructed $\pi^{0}$ momentum spectra of $\mathrm{NC} \pi^{0}$ samples (a), and reconstructed gamma energy spectrum of $\mathrm{NC} \gamma$-deexcitation sample (b).

INGRID throughout the whole data taking period. At the peak energy $0.6 \mathrm{GeV}$, the (anti)neutrino mode beam contains $97.2 \%(96.2 \%) \stackrel{(-)}{\nu}_{\mu}$, with only $0.42 \%(0.46 \%)$ $\stackrel{(-)}{\nu}$ contamination, and the flux uncertainty is approximately $9 \%$.

Neutrino events at the near and far detectors are generated by the NEUT 5.3.2 neutrino interaction generator 60]], which accounts for general interaction and crosssection effects. Most of the cross-section and neutrino flux parameters are constrained by ND280. The unoscillated CC candidate events at ND280 are classified into different samples according to the event topology and are fit with a binned Poisson likelihood to extract the best-fit parameters and correlated uncertainties. The central values and their covariances are then propagated to estimate the far detector flux and cross-section parameters and uncertainty covariance matrix. The fit to ND data was done assuming no oscillation at ND280. This approximation is valid for small $\Delta m_{41}^{2}$, below around $0.3 \mathrm{eV}^{2} / \mathrm{c}^{4}$. However, $\mathrm{NC}$ and $\nu_{e}$ interaction parameters are not constrained by the ND280 fit. As a result, an additional uncorrelated $30 \%$ normalization uncertainty is used in this analysis for the $\mathrm{NC} 1 \pi$ and NCQE channels. The values of these uncertainties are conservative estimates determined from a previous crosssection analysis [61] and NCQE theoretical model comparisons [62,63]. They therefore dominate the overall cross-section uncertainty in the NC oscillation samples. 
At the far detector, there are NEUT parameters (present in ND280 fit but not constrained) that control the final state interactions inside nuclei and secondary interactions with water molecules, altering the event topologies of outgoing particles. For each event topology, the SK selection efficiency and misidentification rate is separately parametrized. The NC $\gamma$-deexcitation sample has separate uncertainties related to primary and secondary $\gamma$ production [49]. The uncertainties in these parameters constitute the SK detector uncertainties.

The effects of systematic uncertainties on the predicted event rates are summarized in Table II. The NC crosssection errors are dominant in the NC samples.

Using the flux and cross-section inputs from ND280, the unoscillated event sample spectra at the far detector are calculated. Oscillation parameters are varied to obtain the best agreement between data and predicted event rates. A joint maximum-likelihood fit to eight far detector samples constrains the sterile mixing parameters $\sin ^{2} \theta_{24}$, $\sin ^{2} \theta_{34}$ and $\Delta m_{41}^{2}$. The log-likelihood is defined as

$-\ln \mathcal{L}=\sum_{i}\left[\mu_{i}-n_{i}+n_{i} \ln \left(n_{i} / \mu_{i}\right)\right]+\frac{1}{2} \Delta \vec{f}^{T} V^{-1} \Delta \vec{f}$,

where $n_{i}$ is the number of events in the $i$ th data bin, and $\mu_{i}=\mu_{i}(\vec{\theta}, \vec{f})$ is the expected event rate with oscillation parameters $\vec{\theta}$ and systematic parameters $\vec{f}$. The last term in Eq. (7) accounts for the systematic penalty with $\Delta \vec{f}$ being the difference between the systematic parameters and their prior values, related by the covariance matrix $V$. The oscillation parameters $\sin ^{2} \theta_{23},\left|\Delta m_{32}^{2}\right|$ and $\delta_{C P}$ are allowed to vary without constraint; $\theta_{12}$ and $\Delta m_{21}^{2}$ are fixed to their PDG values [48]; and a penalty term is used to constrain $\sin ^{2} 2 \theta_{13}=0.0857 \pm 0.0046$.

During the fitting process, at a grid point of $\left(\sin ^{2} \theta_{24}, \sin ^{2} \theta_{34}, \Delta m_{41}^{2}\right)$, the function in Eq. (7) is minimized with respect to the other oscillation parameters and systematic parameters. We use Wilks's theorem to estimate the confidence levels (C.L.) [64]. The results are crosschecked with Gaussian $\mathrm{CL}_{\mathrm{s}}$ contours [65] to ensure no

TABLE II. Percentage systematic uncertainty on far detector event yields.

\begin{tabular}{lcccr}
\hline \hline Sample & Flux & Cross section & SK detector & Total \\
\hline$\nu_{\mu} \mathrm{CC}-0 \pi$ & 4.1 & 4.7 & 3.3 & 4.8 \\
$\bar{\nu}_{\mu} \mathrm{CC}-0 \pi$ & 3.8 & 4.0 & 2.9 & 4.1 \\
$\nu_{e} \mathrm{CC}-0 \pi$ & 4.3 & 5.5 & 3.8 & 6.4 \\
$\bar{\nu}_{e} \mathrm{CC}-0 \pi$ & 3.9 & 5.2 & 4.3 & 6.4 \\
$\nu_{e} \mathrm{CC}-1 \pi^{+}$ & 4.3 & 5.0 & 17.1 & 17.7 \\
$\nu \mathrm{NC} \pi^{0}$ & 4.2 & 20.1 & 8.8 & 21.3 \\
$\bar{\nu} \mathrm{NC} \pi^{0}$ & 3.8 & 19.1 & 8.6 & 20.4 \\
$\mathrm{NC} \gamma$-deexcit & 4.1 & 21.1 & 13.2 & 23.3 \\
\hline \hline
\end{tabular}

significant bias due to the physical limit of $\sin ^{2} \theta_{24} \geq 0$ and $\sin ^{2} \theta_{34} \geq 0$.

\section{RESULTS}

We consider the parameter space of $\Delta m_{41}^{2}>\Delta m_{21}^{2}$ which is most sensitive in T2K. Two categories of fits are done for neutrino mass normal hierarchy $(\mathrm{NH}$, $\left.\Delta m_{31}^{2}>0\right)$ and inverted hierarchy $\left(\mathrm{IH}, \Delta m_{31}^{2}<0\right)$ respectively. The case of $\Delta m_{41}^{2}<0$ is very similar and can be obtained by flipping the hierarchy. The " $3+1$ " best fit differs from the standard three flavor best fit by $\Delta \chi^{2}=1.0$ (4.7) for $\mathrm{NH}(\mathrm{IH})$. From 2500 sets of MC studies with statistical fluctuations, this level of disagreement is expected with the standard three flavor hypothesis in $50 \%(30 \%)$ of the studies.

In the $\left(\sin ^{2} \theta_{24}, \Delta m_{41}^{2}\right)$ parameter plane, $\sin ^{2} \theta_{24}$ is scanned from $10^{-3}$ to 1 , and $\Delta m_{41}^{2}$ from $10^{-4}$ to $0.3 \mathrm{eV}^{2} / c^{4}$. For larger values of $\Delta m_{41}^{2}$, oscillations would also be seen at the near detectors, which is beyond the scope of this analysis. Figure 3 shows the T2K 90\% exclusion limits together with results from other experiments. We have set the most stringent limit on $\sin ^{2} \theta_{24}$ for $\Delta m_{41}^{2}<3 \times 10^{-3} \mathrm{eV}^{2} / c^{4}$. In particular, the NC samples improve the limit by around $20 \%$ for $\Delta m_{41}^{2}<10^{-3} \mathrm{eV}^{2} / c^{4}$. The limit is weaker at larger $\Delta m_{41}^{2}$ due to the lack of high energy events, resulting from the sharply peaked off-axis neutrino flux. The difference between $\mathrm{NH}$ and $\mathrm{IH}$ comes from the $\Delta m_{43}^{2}$ oscillation term. It becomes particularly important when $\Delta m_{41}^{2} \sim \Delta m_{31}^{2}$, as this results in very different values of $\Delta m_{43}^{2}$ in $\mathrm{NH}$ and IH. In partly degenerate

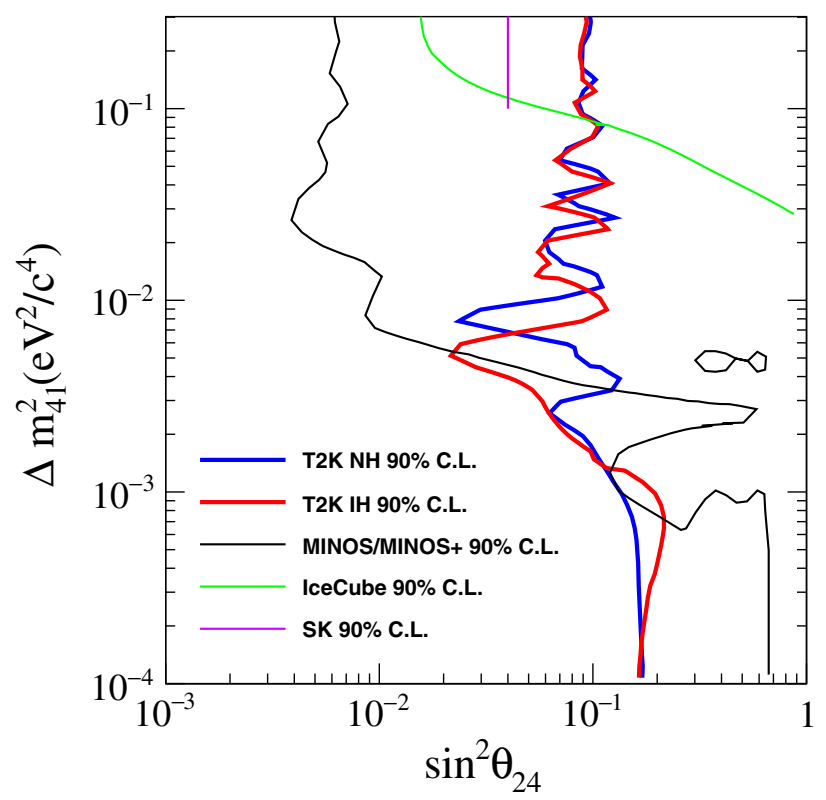

FIG. 3. The T2K $90 \%$ exclusion limits on $\sin ^{2} \theta_{24}$ as a function of $\Delta m_{41}^{2}$, with results from other experiments [35,37,38]. The areas on the right are excluded. 

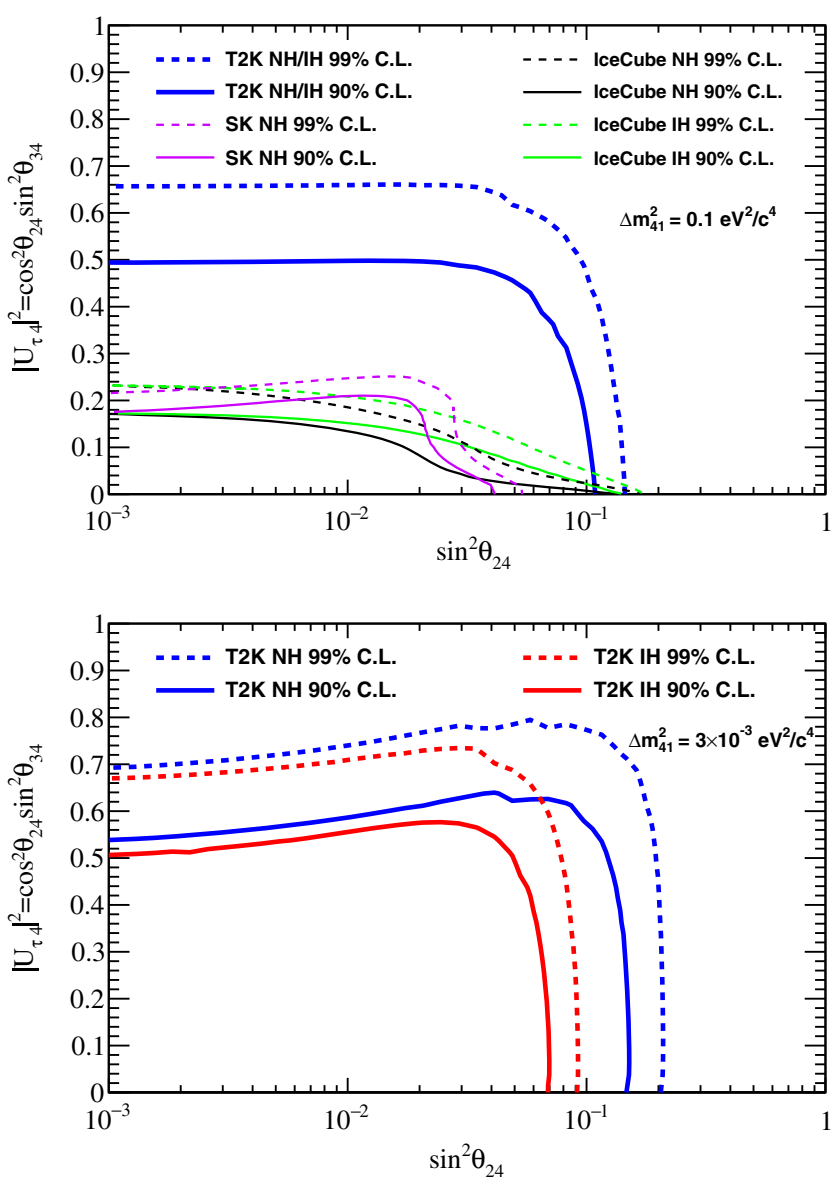

FIG. 4. The T2K $90 \%$ and $99 \%$ exclusion limits on $\sin ^{2} \theta_{24}$ and $\left|U_{\tau 4}\right|^{2}=\cos ^{2} \theta_{24} \sin ^{2} \theta_{34}$ at $\Delta m_{41}^{2}=0.1 \mathrm{eV}^{2}$ (top), with results from other experiments [37,39], and exclusion limits at $\Delta m_{41}^{2}=$ $3 \times 10^{-3} \mathrm{eV}^{2}$ (bottom). The areas on the right are excluded.

cases where $\Delta m_{41}^{2}$ and $\Delta m_{31}^{2}$ are in integer multiples, the $\stackrel{(-)}{\nu}_{e}$ and $\mathrm{NC}$ samples are important in resolving ambiguities. In cases where one of the $\Delta m^{2}$ values is very small, matter effects can significantly alter which mass states are involved in the oscillation, but the overall $\stackrel{(-)}{\nu}_{e}$ appearance probability is not affected by more than a few percent so this does not significantly modify the exclusion limits.

The NC samples allow us to constrain $\theta_{34}$ in conjunction with $\theta_{24}$. Because these samples have low statistics and large cross-section uncertainties, we have limited sensitivity, but our results are consistent with other measurements. Figure 4 shows that we constrain $\sin ^{2} \theta_{24}<0.1$ and $\left|U_{\tau 4}\right|^{2}=\cos ^{2} \theta_{24} \sin ^{2} \theta_{34}<0.5$ at $90 \%$ C.L. if $\Delta m_{41}^{2}=$ $0.1 \mathrm{eV}^{2} / c^{4}$ is assumed. At smaller $\Delta m_{41}^{2}$ values, the limits are different between $\mathrm{NH}$ and $\mathrm{IH}$.

\section{CONCLUSIONS}

Data collected by the T2K experiment between 2010 and 2017 (T2K Runs 1-8) have been used to search for oscillation signatures due to light sterile neutrinos in the " $3+1$ " model. The sterile mixing parameters $\left(\sin ^{2} \theta_{24}, \sin ^{2} \theta_{34}, \Delta m_{41}^{2}\right)$ are constrained by performing a joint fit of the five $\mathrm{CC}$ samples $\stackrel{(-)}{\nu}_{\mu} \mathrm{CC}-0 \pi, \stackrel{(-)}{\nu}_{e} \mathrm{CC}-0 \pi$, and $\nu_{e} \mathrm{CC}-1 \pi^{+}$and the three new $\mathrm{NC}$ samples $\stackrel{(-)}{\nu} \mathrm{NC} \pi^{0}$, $\mathrm{NC}$ $\gamma$-deexcitation, selected at the far detector. Systematic uncertainties on the neutrino flux and CC interaction cross section are constrained by the ND280 data, while NC cross-section uncertainties are determined from a comparison of theoretical models and external data. The data are consistent with the standard three flavor oscillation hypothesis. Limits have been set on the sterile mixing parameters, with the world's best constraint on $\sin ^{2} \theta_{24}$ for $10^{-4} \mathrm{eV}^{2} / c^{4}<\Delta m_{41}^{2}<3 \times 10^{-3} \mathrm{eV}^{2} / c^{4}$. The data related to the measurement and results presented in this paper can be found in [66].

Our current precision is restricted by statistics and the uncertainty on the NC interaction cross section. Apart from future updates of the analysis as we take more data, dedicated systematic studies are required for further improvements to the precision. Another possible extension is to perform a joint analysis of near and far detector data that would expand the range of constraint to $\Delta m_{41}^{2} \gtrsim 1 \mathrm{eV}^{2} / c^{4}$ with additional data at smaller $L / E$.

\section{ACKNOWLEDGMENTS}

We thank the J-PARC staff for superb accelerator performance. We thank the CERN NA61/SHINE Collaboration for providing valuable particle production data. We acknowledge the support of MEXT, Japan; NSERC (Grant No. SAPPJ-2014-00031), NRC and CFI, Canada; CEA and CNRS/IN2P3, France; DFG, Germany; INFN, Italy; National Science Centre (NCN) and Ministry of Science and Higher Education, Poland; RSF, RFBR, and MES, Russia; MINECO and ERDF funds, Spain; SNSF and SERI, Switzerland; STFC, UK; and DOE, USA. We also thank CERN for the UA1/ NOMAD magnet, DESY for the HERA-B magnet mover system, NII for SINET4, the WestGrid and SciNet consortia in Compute Canada, and GridPP in the United Kingdom. In addition, participation of individual researchers and institutions has been further supported by funds from ERC (FP7), "la Caixa" Foundation (ID 100010434, fellowship code LCF/BQ/IN17/11620050), the European Union's Horizon 2020 Research and Innovation programme under the Marie Sklodowska-Curie grant agreement no. 713673 and H2020 Grant No. RISE-GA644294JENNIFER 2020; JSPS, Japan; Royal Society, UK; the Alfred P. Sloan Foundation and the DOE Early Career program, USA. 
[1] B. T. Cleveland, T. Daily, R. Davis, Jr., J. R. Distel, K. Lande, C. K. Lee, P. S. Wildenhain, and J. Ullman, Astrophys. J. 496, 505 (1998).

[2] J. N. Abdurashitov et al. (SAGE Collaboration), Phys. Rev. C 80, 015807 (2009).

[3] M. Altmann et al. (GNO Collaboration), Phys. Lett. B 616, 174 (2005).

[4] W. Hampel et al. (GALLEX Collaboration), Phys. Lett. B 447, 127 (1999).

[5] B. Aharmim et al. (SNO Collaboration), Phys. Rev. C 72, 055502 (2005).

[6] K. Abe et al. (Super-Kamiokande Collaboration), Phys. Rev. D 83, 052010 (2011).

[7] T. Araki et al. (KamLAND Collaboration), Phys. Rev. Lett. 94, 081801 (2005).

[8] F. P. An et al. (Daya Bay Collaboration), Phys. Rev. Lett. 108, 171803 (2012).

[9] Y. Abe et al. (Double Chooz Collaboration), Phys. Lett. B 723, 66 (2013).

[10] J. K. Ahn et al. (RENO Collaboration), Phys. Rev. Lett. 108, 191802 (2012).

[11] M. H. Ahn et al. (K2K Collaboration), Phys. Rev. D 74, 072003 (2006).

[12] P. Adamson et al. (MINOS Collaboration), Phys. Rev. Lett. 110, 251801 (2013).

[13] K. Abe et al. (T2K Collaboration), Phys. Rev. Lett. 112, 181801 (2014).

[14] N. Agafonova et al. (OPERA Collaboration), Phys. Rev. D 89, 051102 (2014).

[15] P. Adamson et al. (NOvA Collaboration), Phys. Rev. D 93, 051104 (2016).

[16] Y. Fukuda et al. (Super-Kamiokande Collaboration), Phys. Rev. Lett. 81, 1562 (1998).

[17] M. G. Aartsen et al. (IceCube Collaboration), Phys. Rev. D 91, 072004 (2015).

[18] B. Pontecorvo, Sov. Phys. JETP 26, 984 (1968) [Zh. Eksp. Teor. Fiz. 53, 1717 (1967)].

[19] V. Gribov and B. Pontecorvo, Phys. Lett. 28B, 493 (1969).

[20] Z. Maki, M. Nakagawa, and S. Sakata, Prog. Theor. Phys. 28, 870 (1962).

[21] A. Aguilar et al. (LSND Collaboration), Phys. Rev. D 64, 112007 (2001).

[22] A. A. Aguilar-Arevalo et al. (MiniBooNE Collaboration), Phys. Rev. Lett. 110, 161801 (2013).

[23] A. A. Aguilar-Arevalo et al. (MiniBooNE Collaboration), Phys. Rev. Lett. 121, 221801 (2018).

[24] J. N. Bahcall, P. I. Krastev, and E. Lisi, Phys. Lett. B 348, 121 (1995).

[25] J. N. Abdurashitov et al., Phys. Rev. C 73, 045805 (2006).

[26] G. Mention, M. Fechner, T. Lasserre, T. A. Mueller, D. Lhuillier, M. Cribier, and A. Letourneau, Phys. Rev. D 83, 073006 (2011).

[27] S. M. Bilenky, C. Giunti, and W. Grimus, Prog. Part. Nucl. Phys. 43, 1 (1999).

[28] V. D. Barger, B. Kayser, J. Learned, T. J. Weiler, and K. Whisnant, Phys. Lett. B 489, 345 (2000).

[29] J. Kopp, P. A. N. Machado, M. Maltoni, and T. Schwetz, J. High Energy Phys. 05 (2013) 050.

[30] S. Gariazzo, C. Giunti, M. Laveder, Y. F. Li, and E. M. Zavanin, J. Phys. G 43, 033001 (2016).
[31] S. Schael et al. (SLD Electroweak Group, DELPHI, ALEPH, SLD, SLD Heavy Flavour Group, OPAL, LEP Electroweak Working Group, L3 Collaboration), Phys. Rep. 427, 257 (2006).

[32] I. E. Stockdale et al., Phys. Rev. Lett. 52, 1384 (1984).

[33] G. Cheng et al. (SciBooNE and MiniBooNE Collaboration), Phys. Rev. D 86, 052009 (2012).

[34] K. Abe et al. (T2K Collaboration), Phys. Rev. D 91, 051102 (2015).

[35] P. Adamson et al. (MINOS+ Collaboration), Phys. Rev. Lett. 122, 091803 (2019).

[36] P. Adamson et al. (NOvA Collaboration), Phys. Rev. D 96, 072006 (2017).

[37] K. Abe et al. (Super-Kamiokande Collaboration), Phys. Rev. D 91, 052019 (2015).

[38] M. G. Aartsen et al. (IceCube Collaboration), Phys. Rev. Lett. 117, 071801 (2016).

[39] M. G. Aartsen et al. (IceCube Collaboration), Phys. Rev. D 95, 112002 (2017).

[40] H. Harari and M. Leurer, Phys. Lett. B 181, 123 (1986).

[41] P. Adamson et al. (Daya Bay and MINOS Collaborations), Phys. Rev. Lett. 117, 151801 (2016).

[42] M. Wallraff and C. Wiebusch, Comput. Phys. Commun. 197, 185 (2015).

[43] K. Abe et al., Nucl. Instrum. Methods Phys. Res., Sect. A 659, 106 (2011).

[44] S. Fukuda et al., Nucl. Instrum. Methods Phys. Res., Sect. A 501, 418 (2003).

[45] K. Abe et al. (T2K Collaboration), Nucl. Instrum. Methods Phys. Res., Sect. A 694, 211 (2012).

[46] K. Abe et al. (T2K Collaboration), Phys. Rev. D 91, 072010 (2015).

[47] K. Abe et al. (T2K Collaboration), Phys. Rev. Lett. 121, 171802 (2018).

[48] C. Patrignani et al. (Particle Data Group), Chin. Phys. C 40, 100001 (2016).

[49] K. Abe et al. (T2K Collaboration), Phys. Rev. D 90, 072012 (2014).

[50] D. Rein and L. M. Sehgal, Ann. Phys. (N.Y.) 133, 79 (1981).

[51] D. Rein and L. M. Sehgal, Nucl. Phys. B223, 29 (1983).

[52] O. Benhar, N. Farina, H. Nakamura, M. Sakuda, and R. Seki, Phys. Rev. D 72, 053005 (2005).

[53] O. Benhar, A. Fabrocini, S. Fantoni, and I. Sick, Nucl. Phys. A579, 493 (1994).

[54] R. Bradford, A. Bodek, H. S. Budd, and J. Arrington, Nucl. Phys. B, Proc. Suppl. 159, 127 (2006).

[55] A. M. Ankowski, O. Benhar, T. Mori, R. Yamaguchi, and M. Sakuda, Phys. Rev. Lett. 108, 052505 (2012).

[56] K. Abe et al. (Super-Kamiokande Collaboration), Phys. Rev. D 83, 052010 (2011).

[57] K. Abe et al. (T2K Collaboration), Phys. Rev. D 96, 092006 (2017).

[58] K. Abe et al. (T2K Collaboration), Phys. Rev. D 87, 012001 (2013).

[59] N. Abgrall et al. (NA61/SHINE Collaboration), Eur. Phys. J. C 76, 84 (2016).

[60] Y. Hayato, Acta Phys. Pol. B 40, 2477 (2009). 
[61] K. Abe et al. (T2K Collaboration), Phys. Rev. D 91, 072010 (2015).

[62] A. M. Ankowski, M. B. Barbaro, O. Benhar, J. A. Caballero, C. Giusti, R. González-Jiménez, G. D. Megias, and A. Meucci, Phys. Rev. C 92, 025501 (2015).

[63] A. M. Ankowski, AIP Conf. Proc. 1663, 080002 (2015).

[64] S. S. Wilks, Ann. Math. Stat. 9, 60 (1938).
[65] X. Qian, A. Tan, J. J. Ling, Y. Nakajima, and C. Zhang, Nucl. Instrum. Methods Phys. Res., Sect. A 827, 63 (2016).

[66] K. Abe et al. (T2K Collaboration), http://t2k-experiment .org/results/t2kdata-sterile-2017/.

Correction: A curve in Figure 3 and the related Ref. [35] were out of date and required revision. 